\title{
Development of Bone Canaliculi During Bone Repair
}

\author{
K. KUSUZAKI,${ }^{1}$ N. KAGEYAMA,${ }^{1}$ H. SHINJO, ${ }^{1}$ H. TAKESHITA,${ }^{1}$ H. MURATA, ${ }^{1}$ S. HASHIGUCHI, ${ }^{1}$ \\ T. ASHIHARA, ${ }^{2}$ and Y. HIRASAWA ${ }^{1}$
}

Departments of ${ }^{1}$ Orthopaedic Surgery and ${ }^{2}$ Pathology, Kyoto Prefectural University of Medicine, Kyoto, Japan

We recently found that silver impregnation staining with protargol (silver protein), that is, a modified Bodian method, is useful for histologically identifying the details of bone canaliculi structure, using thin sections of decalcified bone tissues. With this staining method, we conducted the present study to assess the development of bone canaliculi during the process of intramembranous ossification using a fracture-like stimulation model of the rat femur. After making a drill-hole in the cortex of the rat femur, decalcified thin sections were obtained after $3,5,7$, and 14 days by the standard paraffinembedding procedure. Silver staining for bone canaliculi was performed using our previously reported technique. The results showed that woven bone covered the fracture surface of the cortex after 5 days, then immature lamellar bone attached to the woven bone after 7 days, and finally the lamellar bone matured and became thick with appositional growth after 14 days. The osteocytes in the woven bone appeared at an early stage of bone repair and developed a few canaliculi that were short and irregularly distributed in the osteoid matrix, while the osteocytes in the lamellar bone at a late stage formed many bone canaliculi that were long and regularly distributed in mature bone matrix. Therefore, we concluded that woven bone osteocytes may be necessary for induction of the lamellar bone osteocytes followed by active appositional growth of the lamellar bone at the early stage of bone repair, and also that both bone tissues could be clearly distinguished from one another based on the pattern of development of bone canaliculi by the osteocytes, as seen with the use of our sensitive staining method. (Bone 27: 655-659; 2000) (c) 2000 by Elsevier Science Inc. All rights reserved.

Key Words: Bone canaliculi; Osteocytes; Bone repair; Intramembranous ossification; Woven bone; Lamellar bone; Silver staining.

\section{Introduction}

It has been difficult to clearly observe bone canaliculi using decalcified thin sections of paraffin-embedded materials via the standard method. ${ }^{1,2,5,11,12,19}$ Recently, we reported that silver impregnation staining with protargol (silver protein), that is, a modified Bodian method, ${ }^{3,4}$ was useful for morpho-

Address for correspondence and reprints: Dr. Katsuyuki Kusuzaki, Department of Orthopaedic Surgery, Kyoto Prefectural University of Medicine, Kamigyo-ku Kawaramachi Hirokoji, Kyoto 602-8566, Japan. E-mail: kusu@basic.kpu-m.ac.jp logical demonstration of the bone canaliculi structure, even when using decalcified bone tissues. ${ }^{13}$ In that report, we demonstrated the details of the fine structures of numerous bone canaliculi in rat cortical bone or intramedullary cancellous or woven bone at the metaphysis. In this study, using our staining method, we investigated the development of bone canaliculi during the process of intramembranous ossification using a fracture-like stimulation model of the rat femur.

\section{Materials and Methods}

Under anesthesia with ether, a drill-hole was made in the cortex of the femoral shaft of 16 rats (Wistar strain, male, mean age 5-6 weeks, mean body weight $150 \mathrm{~g}$ ) with an $18 \mathrm{G}$ needle, according to a previously reported technique (Figure 1) ${ }^{18}$ On days 3, 5, 7, and 14 , the femora were excised in a separate group (four rats each) after killing with ether. The bones were fixed in $10 \%$ buffered formalin immediately after excision for 5 days, and were then decalcified in $20 \%$ buffered ethylene-diamine tetraacetic acid (EDTA) solution for 3 weeks at room temperature. After trimming, the bones were embedded in paraffin by the standard method. Sections of 4-6 $\mu \mathrm{m}$ were mounted on glass slides coated with gelatin film to prevent detachment of the sections from the slides.

After deparaffinization by xylene and ethanol, the sections were rinsed with double-distilled water (DDW) at room temperature. These sections were then stained with $2 \%$ protargol (BDH Co., UK) solution containing copper beads $(7.5 \mathrm{~g}$ to $40 \mathrm{~mL}$ staining solution) for $48 \mathrm{~h}$ at $37^{\circ} \mathrm{C}$. The reduction of silver in the sections was accomplished with a solution containing hydroqui-

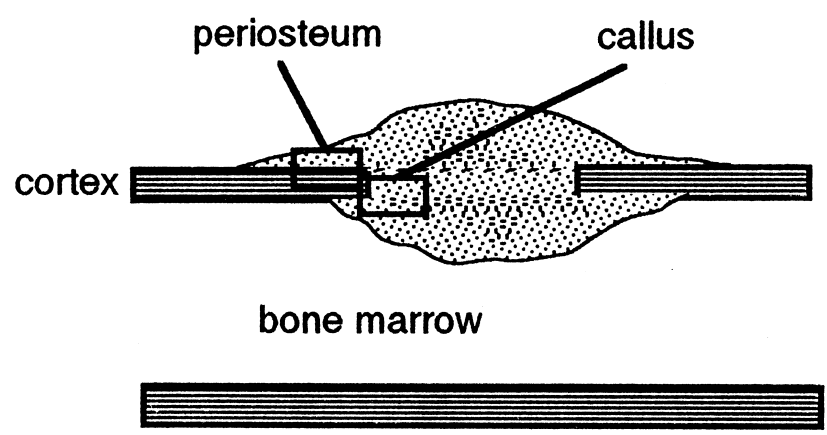

Figure 1. Illustration of the experimental fracture repair model by the drill-hole method performed in the present study. After drilling using an $18 \mathrm{G}$ needle, the callus and periosteum in contact with the cortex were sampled on days $3,5,7$, and 14 . 


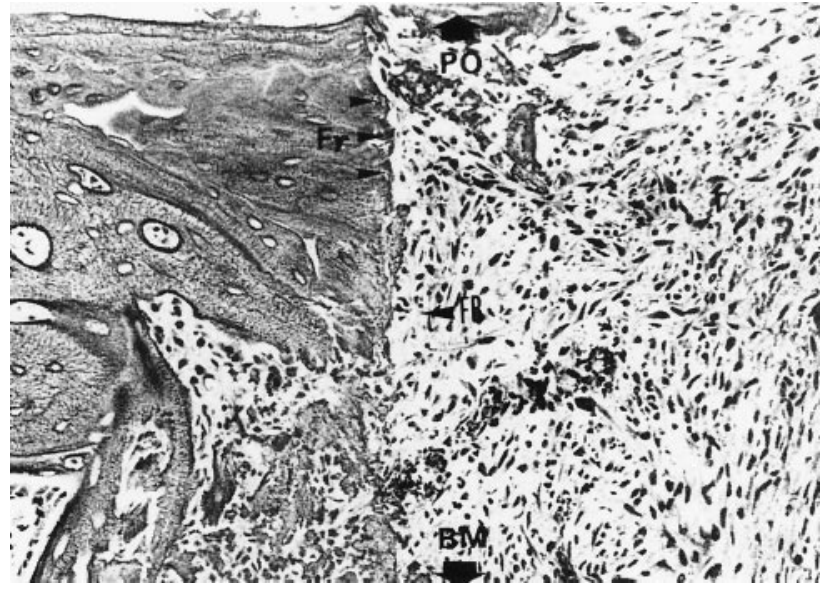

Figure 2. Histological findings of the callus stained with protargol on day 3 after drilling (original magnification $\times 10$ of an objective lens in original). Many fibroblastic mesenchymal cells (FB) with blood vessels are seen around the fracture end ( $\mathrm{Fr}$ with arrowheads). At this point these cells have neither produced bone matrix nor developed any bone canaliculi. The upper region of the figure is periosteum (PO), and the lower region is bone marrow $(\mathrm{BM})$.

none and formalin (1 g hydroquinone, $5 \mathrm{~mL}$ concentrated formalin, and $100 \mathrm{~mL}$ of DDW) for $30 \mathrm{~min}$ at $5^{\circ}-10^{\circ} \mathrm{C}$. After these treatments, the sections were rinsed three times with DDW at room temperature, then treated with a solution of $0.2 \%$ gold chloride for $50 \mathrm{~min}$ at $5^{\circ}-10^{\circ} \mathrm{C}$, and followed with three more rinses with DDW. The color balance of the stained sections was modulated with $2 \%$ oxaluric acid solution for $60 \mathrm{~min}$ at $5^{\circ}-10^{\circ} \mathrm{C}$. Finally, after rinsing with DDW for $5 \mathrm{~min}$, the sections were embedded in balsam after dehydration with ethanol. ${ }^{13}$

To compare the bone matrix findings, the other serial thin sections from each specimen were stained with hematoxylineosin (HE)

All of these studies were performed in accordance with the Declaration of Helsinki and the Interdisciplinary Principles and Guidelines for the Use of Animals in Research, Testing, and Education.

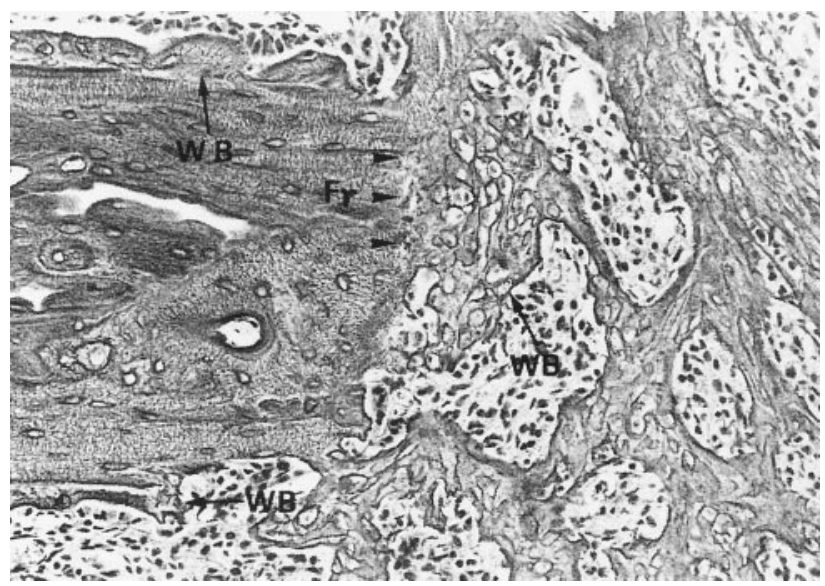

Figure 3. Histological findings of the callus, periosteum, and endosteum around the fracture end $(\mathrm{Fr})$ after 5 days (original magnification $\times 10$ of an objective lens in original, protargol staining). The direction of the section is the same as that shown in the Figure 2. A large amount of woven bone (WB) formed in the callus.

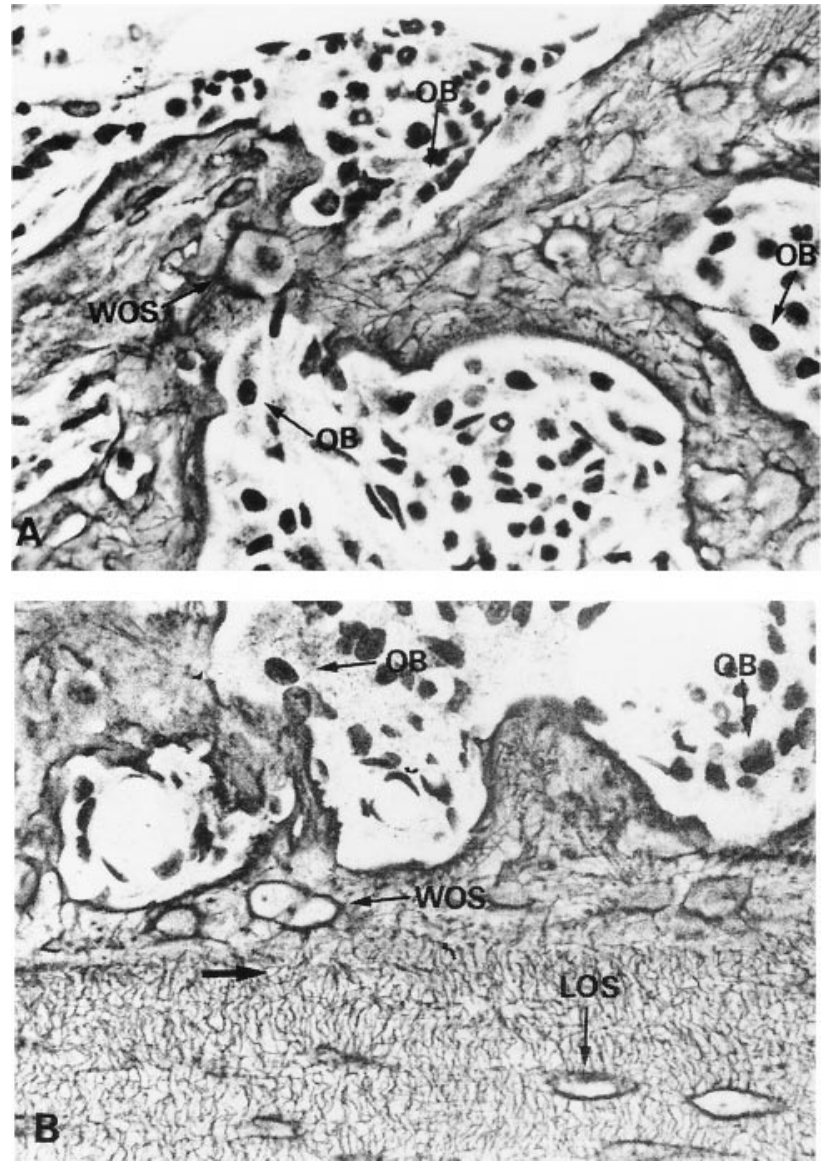

Figure 4. High-magnification photomicrographs of the newly formed woven bone in the callus (A) and periosteum (B), both of which are shown in Figure 3 (original magnification $\times 40$ of an objective lens in original). The woven bone osteocytes (WOS), which appeared at both sites, developed not only fewer but also more irregularly distributed bone canaliculi in the osteoid bone matrix than those of the lamellar bone osteocytes (LOS) of the cortical bone matrix observed in (B). Some of the bone canaliculi of the woven bone osteocytes in the periosteum seem to be connected to those of lamellar bone osteocytes in the cortical bone (arrow).

\section{Results}

At 3 days after bone drilling, many fibroblasts with blood vessels were observed in the callus formed around the drilling site (Figure 2). The osteocytes in the femoral cortex had numerous bone canaliculi distributed regularly. This cortical bone tissue was recognized as mature lamellar bone by HE staining, and therefore these cells were lamellar bone osteocytes. The fracture end of the cortex was covered with fibroblasts that did not produce any bone matrix and had no bone canaliculi.

However, after 5 days, a large amount of newly formed bone matrix appeared, accompanied by many osteoblasts in the callus, covering not only the surface of the fracture site, but also the surrounding periosteum and endosteum (Figure 3). This bone tissue, identified as woven bone by HE staining, was stained reddish-purple by protargol staining. The osteocytes in the newly formed woven bone developed not only fewer but also more irregularly distributed bone canaliculi than the lamellar bone osteocytes (Figure 4A). These cells were considered woven bone osteocytes. Osteoblasts lining the woven bone were stained with protargol. Their nuclei were black, and they had argentaffin 


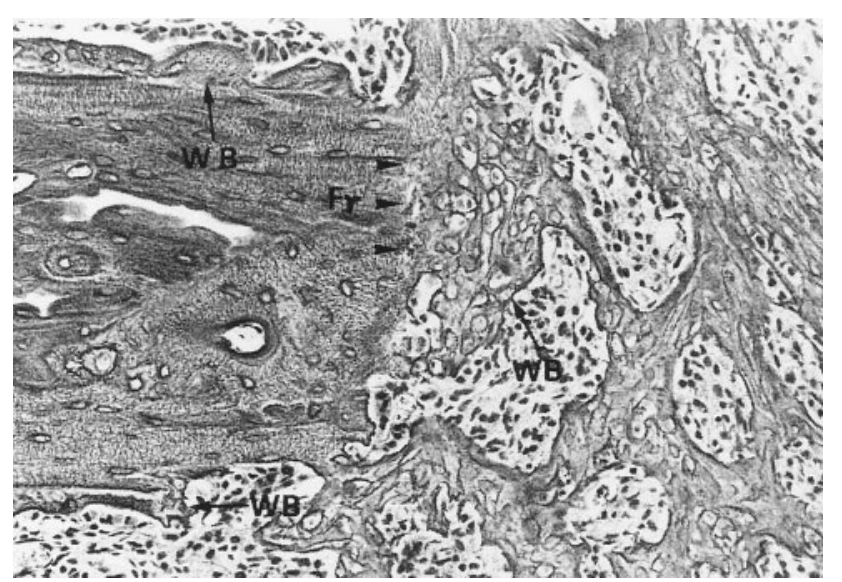

Figure 5. Histological findings of the callus and periosteum around the fracture end ( $\mathrm{Fr}$ ) after 7 days (original magnification $\times 10$ of an objective lens in original). The surface of the woven bone was covered with many immature lamellar bone osteocytes (ILOS) that had regularly distributed bone canaliculi in the bone matrix.
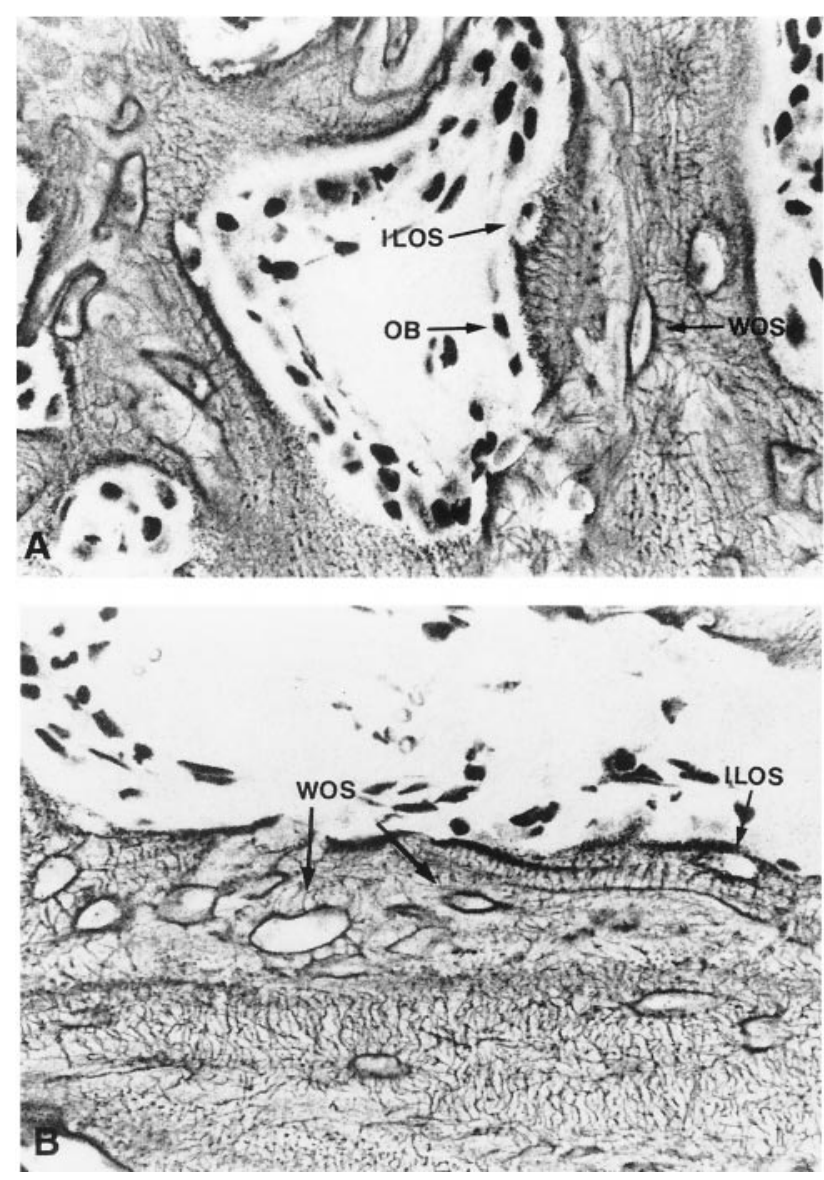

Figure 6. High-magnification photomicrographs of the newly formed lamellar bone in the callus (A) and periosteum (B), both of which are shown in Figure 5 (original magnification $\times 40$ of an objective lens in original). In both sites, immature lamellar bone osteocytes (ILOS) attached to the surface of the woven bone and began to develop regularly distributed bone canaliculi connecting to bone canaliculi of the woven bone osteocytes (WOS) (OB, osteoblasts).
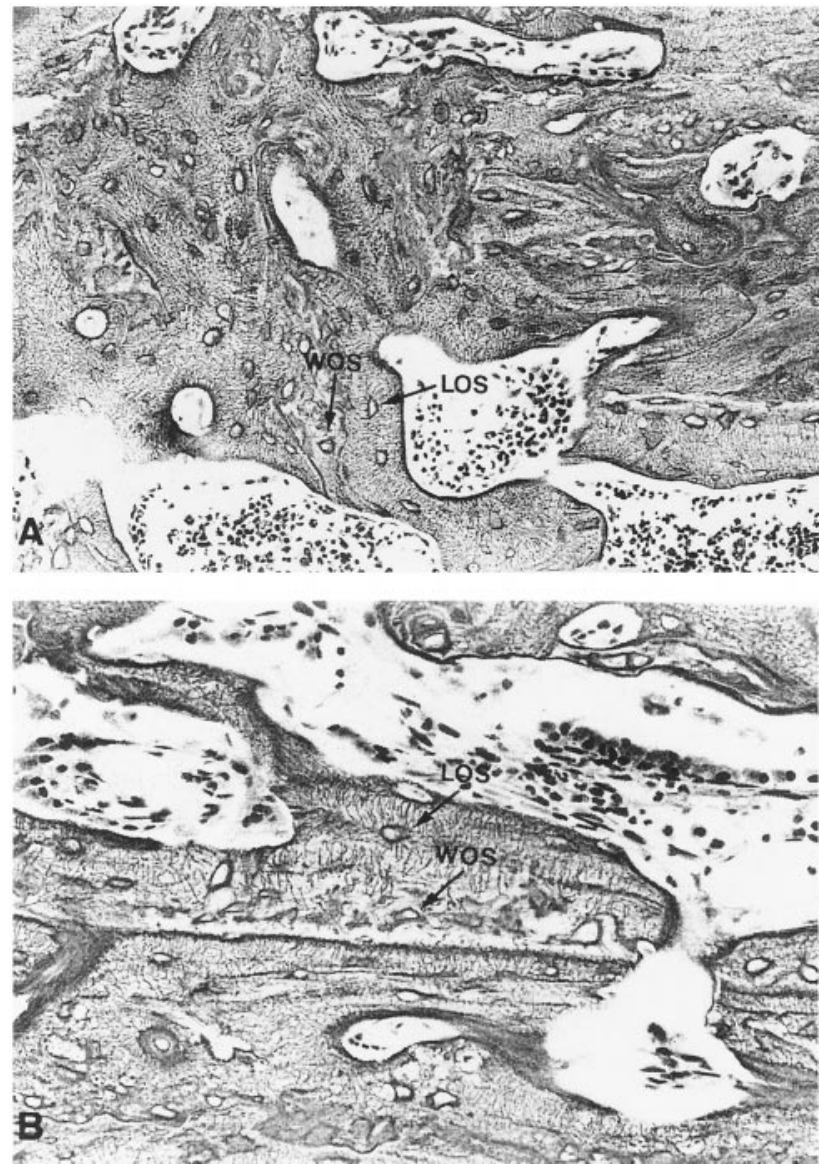

Figure 7. Histological findings of the callus (A) and periosteum (B) around the fracture end (Fr) after 14 days (original magnification $\times 10$ of an objective lens in original). Large amounts of newly formed lamellar bone is seen at both sites. Appositional growth of the lamellar bone was proceeding by the lamellar bone osteocytes (LOS) (WOS, woven bone osteocytes)

granules. The canaliculi of the woven bone osteocytes sometimes seemed to connect to those of the lamellar bone osteocytes in the cortex at the periosteum (Figure 4B).

On day 7 after drilling, the surface of the woven bone around the fracture site was covered with many osteocytes that had many regularly positioned bone canaliculi (Figure 5). These osteocytes attached to the surface of the woven bone matrix at the fracture site as well as at the periosteum and connected their bone canaliculi to those of woven bone osteocytes (Figure 6). Because the pattern of bone canaliculi built up by these osteocytes was more similar to that of the lamellar bone osteocytes than that of woven bone osteocytes, they were considered immature lamellar bone osteocytes.

On day 14, there was a large amount of newly formed mature bone in the callus (Figure 7). The lamellar bone osteocytes developed bone canaliculi and produced more bone matrix. However, woven bone and woven bone osteocytes could still be seen in the callus and periosteum (Figure 8).

\section{Discussion}

The development of bone canaliculi during bone repair has rarely been investigated, ${ }^{18}$ because it has been difficult to morphologically detect bone canaliculi using decalcified paraffin sections, 

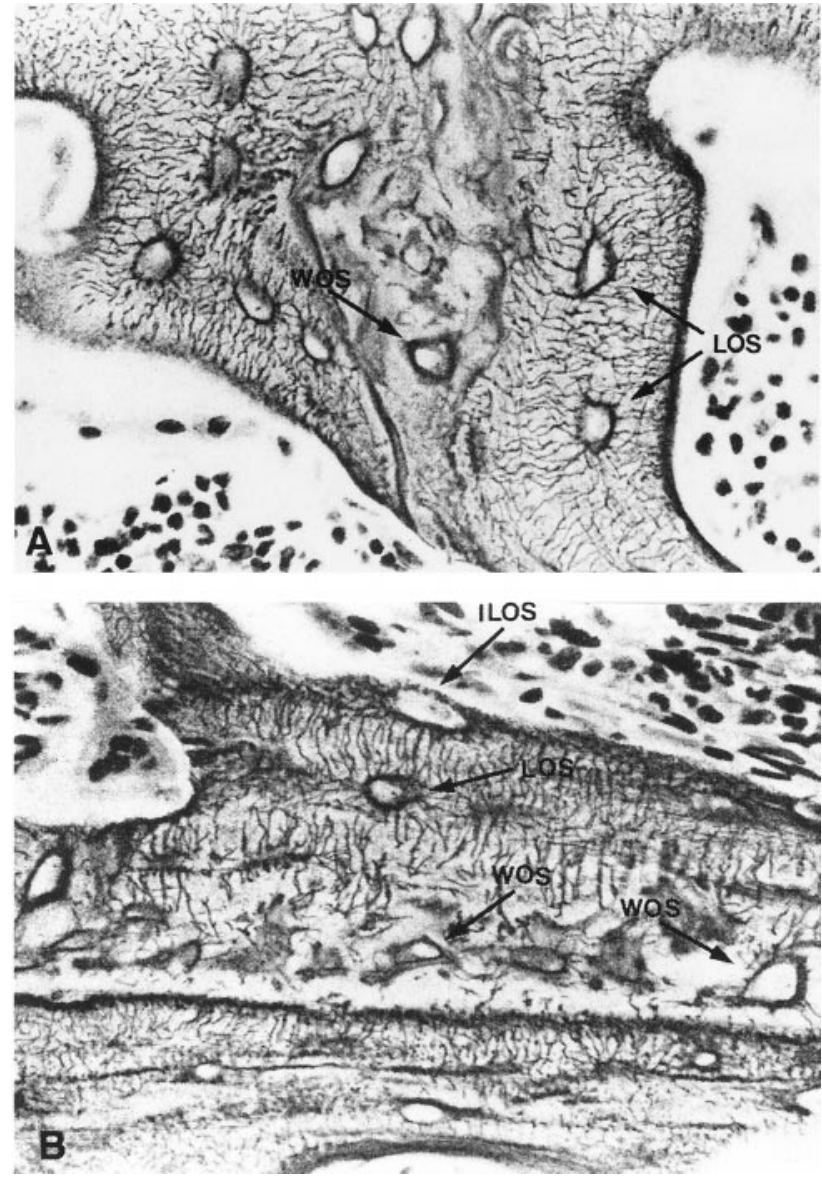

Figure 8. High-magnification photomicrographs of the newly formed lamellar bone in the callus (A) and periosteum (B), both of which are shown in Figure 7 (original magnification $\times 40$ of an objective lens in original). At both sites, the newly formed lamellar bone became thick and immature lamellar bone osteocytes (ILOS) developed bone canaliculi that connected to those of lamellar bone osteocytes (LOS), although the woven bone osteocytes (WOS) still existed.

even when using ground sections ${ }^{1,2,5,11,12,19}$ or electron microscopy. ${ }^{6-10,14-16,18,20,21}$ Most investigators also did not indicate that the lacuna-bone canaliculi system was important for bone formation or bone mechanics, although it is clear that this system plays a significant role in bone mineral metabolism and nutrition of osteocytes..$^{8,10,11,15-17}$ We recently demonstrated that osteocytes can form numerous bone canaliculi in the bone matrix, like the neuron networks in the brain, by the protargol staining technique, using decalcified paraffin sections of bone tissues. ${ }^{13}$ Such a network system of bone canaliculi has never been reported. Using this staining procedure, we also can detect soft tissue cells such as bone marrow, periosteum, endosteum, cartilage, ligament, tendon, vessel, nerve, and muscle. Therefore, the relationship of osteocytes to osteoblasts or vessels can be observed easily, and comparing the stainability of the bone or cartilage matrix with HE was also simple.

The results of the present study reveal that, during bone repair, at an early phase ( 5 days after injury), woven bone osteoblasts derived from bone marrow mesenchymal cells attach to the surface of the injured cortical bone to make a scaffold for the lamellar bone osteoblasts, which differentiate into immature lamellar bone osteocytes, then into mature lamellar osteocytes (Figure 9). By HE staining of decalcified tissues, the woven

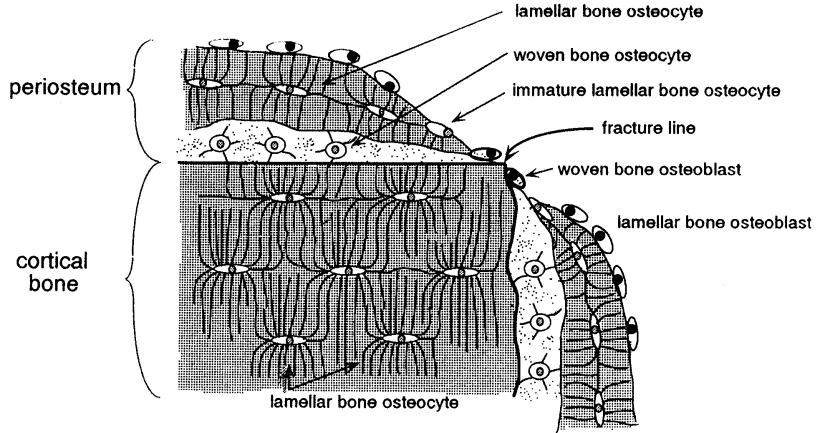

Figure 9. Schematic diagram of bone canaliculi development by woven and lamellar bone osteocytes during bone repair.

bone matrix stained pinkish, whereas the lamellar bone matrix stained reddish, and the shape of the woven bone osteocytes was oval and "plump," whereas that of the lamellar bone osteocytes was spindled and flat. These morphological findings, however,

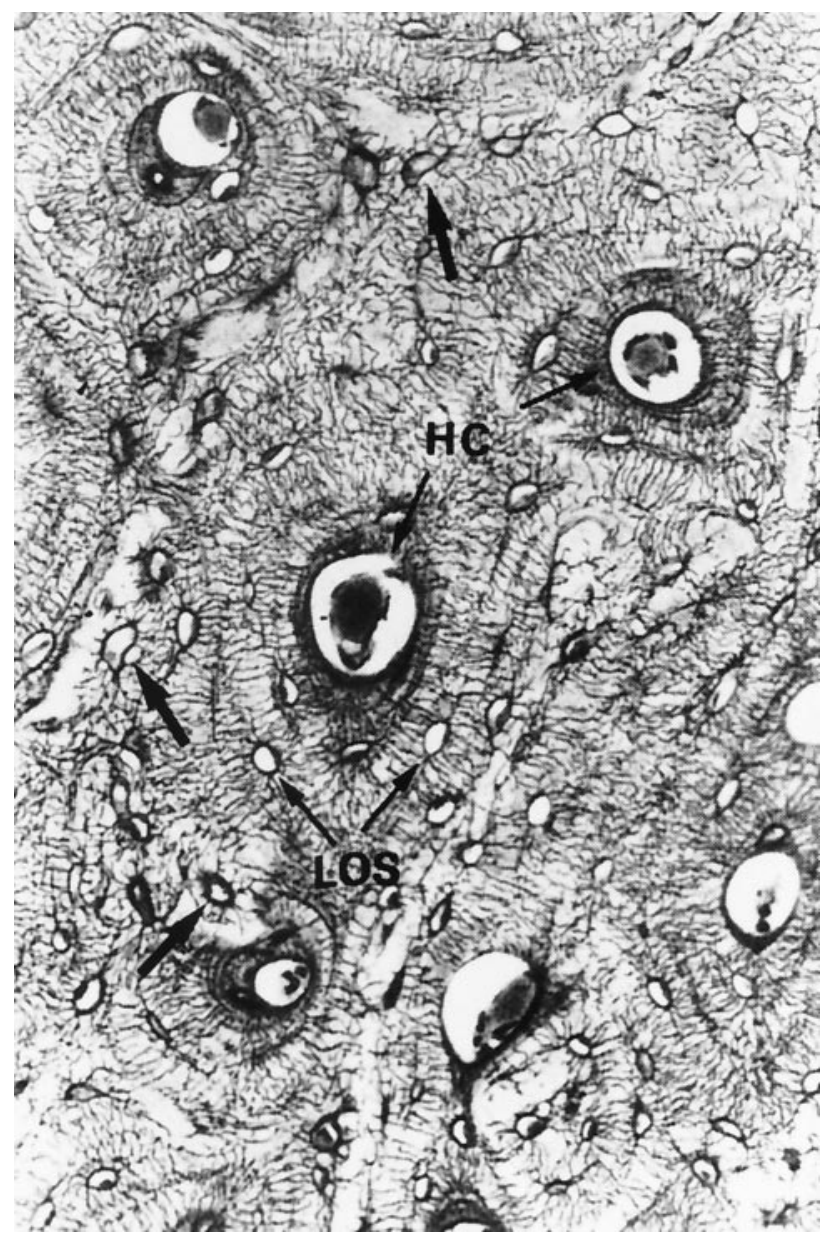

Figure 10. Woven bone osteocytes in the cement line of an osteon. This is a histological picture of a cross-section in the rat femur, stained with protargol after decalcification (original magnification $\times 20$ of an objective lens in original). Most of the lamellar bone osteocytes (LOS) are arranged concentrically around the haversian canal (HC). Their bone canaliculi are distributed, densely and regularly, with regard to both direction of the haversian canal and other osteocytes, but there are some woven bone osteocytes in the cement line of each osteon (thick arrow). 
do not provide adequate evidence in support of a difference in the biological roles of the two matrix types. On the other hand, by protargol staining, although there was only a slight difference in the stainability between the woven and lamellar bone matrices (woven bone stained more reddish than lamellar bone matrix), the pattern of bone canaliculi development was clearly different between the two types. Woven bone osteocytes developed fewer and more irregularly distributed bone canaliculi than lamellar bone osteocytes. Both osteocytes were clearly distinguishable by the difference in development of bone canaliculi. In the periosteum near the fracture end, woven bone osteocytes also appeared, prior to lamellar bone osteocytes. At this site, bone canaliculi of woven bone osteocytes connected to those of the lamellar bone in the cortex. This phenomenon suggests that woven bone osteocytes may supply nutrition to lamellar bone osteocytes and also communicate with them as well as with osteoblasts in bone marrow. Thus, woven bone osteocytes have the capability to attach to the fracture surface at the early phase of bone repair and to induce lamellar bone osteocytes, which is followed by active appositional growth of the lamellar bone at a late phase. The lamellar bone osteocytes probably cannot directly come into contact with the injured bone matrix. Those woven bone osteocytes were found at the metaphysis during endochondral ossification. ${ }^{13}$ At this site, woven bone osteocytes attach to the surface of the growth plate cartilage matrix instead of the fracture surface, then induce active lamellar bone formation, as demonstrated during the bone repair. Therefore, it is suggested that the woven bone osteocytes may be a mediator between the cartilage or injured bone matrix and the lamellar bone during ossification, and are therefore essential for new bone formation. Interestingly, we found woven bone osteocytes in the cement line, which was the outer area of the osteon, as shown in Figure 10. The woven bone osteocytes may also be important for joining osteons together during normal bone formation.

Finally, based on the present results, we conclude that woven bone osteocytes and matrix may be necessary for attachment and maturation of lamellar bone osteocytes at an early stage of bone repair, and also that both bone tissues can be clearly distinguished from each other by the pattern of bone canaliculi development, which was detected by our sensitive protargol staining method.

\section{References}

1. Bancroft, J. D. and Stevens, A. Theory and Practice of Histological Techniques, 2nd Ed. London: Churchill Livingstone 1982; 315-326.

2. Baud, C. A. Submicroscopic structure and functional aspects of the osteocytes Clin Orthop 56:227-236; 1968.
3. Bodian, D. A new method for staining nerve fibers and nerve endings in mounted paraffin section. Anat Rec 65:89-97; 1936.

4. Bodian, D. The staining of paraffin sections of nerve tissue with activated protagol. The role of fixatives. Anat Rec 69:153-162; 1937.

5. Christie, K. N. The demonstration of canaliculi in sections of decalcified bone by silver impregnation method. Stain Technol 52:301-302; 1977.

6. Cooper, R. R. and Milgram, J. W. Morphology of the osteon. An electron microscopic study. J Bone Jt Surg 48-A:1239-1271; 1966.

7. Doty, S. B. Morphological evidence of gap junctions between bone cells. Calcif Tissue Int 33:509-512; 1981.

8. Doty, S. B. and Schofield, B. H. Metabolic and structural changes within osteocytes of rat bone. In: Talmage, R. V. and Munson, P. L., Eds. Calcium, Parathyroid Hormone and Calcitonin. Amsterdam: Exerpta Medica; 1972; 353-364.

9. Ejiri, S. and Ozawa, H. Scanning electron microscopic observations of rat tibia using the HCl-collagenase method. Arch Histol Jpn 45:399-404; 1982.

10. Holtrop, M. E. and Weigner, J. M. Ultrastructural evidence for a transport system in bone. In: Talmage, R. V. and Munson, P. L., Eds. Calcium, Parathyroid Hormone and Calcitonin. Amsterdam: Exerpta Medica; 1972; 365-374.

11. Jee, W. S. S. The skeletal tissue. In: Weiss, L., Ed. Histology, Cell and Tissue Biology. London: Macmillan; 1983; 200-255.

12. Kolliker, A. The osseous system. In: Stricker, S., Ed. Manual of Human Histology. London: Sydenham; 1853; 289-307.

13. Kusuzaki, K., Kageyama, N., Shinjo, H., Murata, H., Takeshita, H., Ashihara, T., and Hirasawa, Y. A staining method for bone canaliculi. Acta Orthop Scand 66:166-168; 1995.

14. Menton, D. N., Simmons, D. J., and Chang, S. L. From bone lining cell to osteocyte-An SEM study. Anat Rec 209:29-39; 1984.

15. Pawlicki, R. Bone canaliculi endings in the area of the osteocyte lacuna. Acta Anat 91:292-304; 1975 .

16. Perry, M. M., John, H. A., and Thomas, N. S. T. Actin-like filaments in the cleavage furrow of newt egg. Exp Cell Res 65:249-253; 1971.

17. Salmon, C. D. and Ray, R. D. The autoradiographic distribution and localization of ${ }^{45} \mathrm{Ca}$ in undecalcified fresh and devitalized rat bone autografts. J Bone Jt Surg 48-A:1575-1584; 1966

18. Shapiro, F. Cortical bone repair. The relationship of the lacuna-canalicular system and intercellular gap junction to the repair process. J Bone Jt Surg 70-A:1067-1081; 1988

19. Tomes, J. Observation on the structure and development of bone. Trans R Soc 143:109-139; 1853.

20. Wasserman, F. and Yaeger, J. A. Fine structure of the osteocyte capsule and of the wall of the lacuna in bone. Z Zellforsh 67:636-652; 1965 .

21. Weinger, J. M. and Holtrop, M. E. An ultrastructural study of bone cells. The occurrence of microtubules, microfilaments and tight junctions. Calcif Tissue Res 14:15-29; 1974.
Date Received: April 27, 2000

Date Revised: June 28, 2000

Date Accepted: July 14, 2000 